\title{
TAXA DE JUROS E PRÊMIO DE RISCO: INVESTIGANDO A HIPÓTESE BRESSER-NAKANO PARA A ECONOMIA BRASILEIRA, 1995-2005*
}

\author{
André M. Marques ${ }^{* *}$ \\ Adelar Fochezatto ${ }^{* *}$
}

RESUMO A proposta Bresser-Nakano deflagrou uma forte discussão sobre a política de juros no Brasil, suscitando uma série de críticas e contribuições para o seu aperfeiçoamento. Seu argumento central baseia-se na suposição de que o sentido da causalidade vai da taxa de juros para o prêmio de risco, e não o contrário. Com isso, a autoridade monetária, ao reduzi-la, promove um círculo virtuoso na economia, reduzindo o prêmio de risco e, através da taxa de câmbio, a própria inflação. Essa hipótese, no entanto, continua controversa e carece de estudos empíricos visando à sua verificação. O objetivo principal deste trabalho é investigar a sua validade para a economia brasileira nos últimos 10 anos de política monetária. Aplicando testes de causalidade de Granger, os resultados encontrados apóiam a hipótese BresserNakano.

Palavras-chave: Bresser-Nakano; integração financeira; taxa de juros; prêmio de risco

Código JEL: E44, F02, F36

\section{INTEREST RATE AND THE RISK PREMIUM: BRESSER-NAKANO'S HYPOTHESIS IN THE BRAZILIAN ECONOMY, 1995-2005}

ABSTRACT The Bresser-Nakano's proposal raised a wide discussion on the monetary policy in Brazil and several critiques were brought about as well as contribu-

* Artigo enviado em 31 de março de 2006 e aprovado em 8 de junho de 2007. Os autores agradecem os valiosos comentários de dois pareceristas anônimos. Eventuais erros e omissões ainda existentes são de responsabilidade dos autores.

** Doutorando do PPGE-UFRGS, bolsista do CNPq, e-mail: 00094751@ufrgs.br

*** Doutor, professor titular do PPGE-PUCRS, pesquisador do CNPq, e-mail: adelar@pucrs.br 
tions for its improvement. Their main argument is based upon a causality relation that runs from interest rate to risk premium with a positive correlation. In this case a cut in the interest rate shifts down the risk premium, and could even reduce the price inflation by the exchange rate. This assumption is linked to others issues in financial integration in an open economy context. The traditional view is that the interest rate is determined by the interest rate parity condition and that the monetary authority has no autonomy to set different rates from those determined by the "capital's flows". An alternative approach defends that is possible to peg with wide autonomy the domestic interest rate, even in this context. The results endorse Bresser-Nakano's proposal and suggest it could be feasible for Brazilian real situation.

Key words: financial integration; interest rate; risk premium 


\section{INTRODUÇÃO}

Nos últimos anos, a economia brasileira tornou-se mais integrada econômica e financeiramente. Nesse novo ambiente, surgiu a questão acerca dos graus de autonomia da política monetária, especialmente da taxa de juros. Essa questão é importante, pois o Brasil possui um estoque expressivo da dívida pública indexada à taxa básica de juros estabelecida pela autoridade monetária. ${ }^{1}$

A visão hegemônica sustenta que, no contexto de abertura e integração financeira, a taxa de juros é determinada pela paridade dos juros internacionais e que, portanto, o Banco Central não tem autonomia para fixá-la em patamares diferentes daqueles definidos pelo mercado. Nos casos em que o Banco Central define a taxa básica, como é o caso do Brasil, esta decorre de uma "função de reação" às expectativas e ao comportamento do mercado, bem como às mudanças nas condições econômicas vigentes (Woodford, 2001; Bogdanski et al., 2000).

Alguns economistas, porém, têm argumentado que, mesmo em ambiente de integração financeira, com perfeita mobilidade de capitais, a autoridade monetária pode fixar a taxa de juros doméstica no nível de sua escolha, favorecendo o crescimento e o emprego, com baixo custo para o capital. ${ }^{2} \mathrm{De}$ acordo com os argumentos de Lavoie (2000, 2001, 2002-3), Smithin (1997, 1999, 2002-3, 2003, cap. 8), Kam e Smithin (2004), Paraskevopoulos et al. (1996) e outros, na medida em que a autoridade monetária impõe uma redução na taxa de juros, reduz o componente risk premium do País e aumenta, concomitantemente, a atratividade de seus títulos no mercado financeiro internacional, impedindo uma maior depreciação da taxa de câmbio. Assim, mesmo em ambiente de perfeita mobilidade de capitais e câmbio flutuante, a autoridade monetária poderia reduzir a taxa de juros doméstica, mantendo a estabilidade de preços, melhorando a solvência das contas públicas ${ }^{3} \mathrm{e}$ obtendo maior crescimento econômico e emprego.

Os argumentos de Bresser e Nakano (2002) vão nessa direção. Segundo eles, o maior desafio da economia brasileira é passar de um equilíbrio instável, e inviável a longo prazo, para um equilíbrio estável. Isso seria alcançado mediante a "(...) iniciativa do Banco Central de anunciar sua decisão de alcançar gradualmente um novo regime de taxa de juros (...)" (Bresser e Nakano, 2002: 167). 
Para esses autores, seria perfeitamente factível que a autoridade monetária no Brasil adotasse os padrões internacionais na definição da taxa de juros, isto é, em níveis similares aos demais países em desenvolvimento (tabela 1). Ao fazer isso, "o Banco Central sinalizará para uma queda gradual e firme da taxa de juros por ele estabelecida. Essa redução de juros, por sua vez, diminuirá, de fato, a probabilidade de default, que, virtuosamente, se refletirá na percepção de risco do financiador" (Ibid., p. 167). Isso porque "uma elevação da taxa interna de juros, qualquer que seja a razão, acabará sendo percebida como um aumento no risco-país e não o contrário" (Bresser e Nakano, 2002: 167).

A hipótese em que se fundamenta o argumento é de que o risco-país estaria positivamente correlacionado com a taxa de juros definida pela autoridade monetária. A redução da taxa de juros e o conseqüente aumento da atratividade dos títulos do Governo no mercado internacional poderiam apreciar a taxa de câmbio e, com isso, até mesmo reduzir a taxa de inflação e melhorar a posição devedora do País.

A proposta Bresser-Nakano desencadeou uma série de trabalhos, em sua maioria criticando seu principal argumento. ${ }^{4}$ A declaração de Oreiro (2002) representa bem a principal objeção à proposta Bresser-Nakano: ele afirmou que se a mesma fosse implantada no Brasil, “(...) sob o atual regime de flutuação cambial, produziria uma forte depreciação da taxa de câmbio e conseqüentemente uma elevação da taxa de inflação" (p. 108).

O objetivo principal deste trabalho é investigar a validade empírica da hipótese central de Bresser e Nakano (2002) de que é factível uma política de redução progressiva da taxa de juros nas condições vigentes do País. Em um segundo momento, o objetivo é verificar algumas de suas implicações mais imediatas sobre o processo de estabilização. Para tanto, procedeu-se a alguns testes de causalidade de Granger, ${ }^{5}$ empregando-se dados mensais para os últimos 10 anos de política econômica no Brasil. As variáveis utilizadas foram a taxa de juros Selic $\left(i_{t}\right)$, fixada pelo Banco Central, e o spread do C-Bond $\left(Z_{t}\right)$ em pontos base mensal, que tem sido utilizado em diversos estudos como o elemento mais representativo da percepção do risco sobre a dívida do País.

Em vista da hipótese, uma redução da taxa de juros também afetaria outras variáveis do sistema. Assim, em um segundo momento, empregan- 
do-se dados mensais e diários para diversos períodos, investigaram-se a precedência temporal e o sentido da correlação entre as seguintes variáveis:

(a) taxa de juros $\left(i_{t}\right)$ e taxa de câmbio nominal $\left(E_{t}\right)$, para averiguar se um aumento da taxa de juros levaria à depreciação da taxa cambial;

(b) taxa de juros $\left(i_{t}\right)$ e taxa de inflação $(\dot{p})$;

(c) variação da taxa de juros $\left(\dot{i}_{t}\right)$ e variação da taxa de câmbio nominal $\left(\dot{e}_{t}\right)$, para avaliar a eventual correlação entre a taxa de juros e a volatilidade da taxa de câmbio;

(d) variação da taxa de câmbio $\left(\dot{E}_{t}\right)$ e taxa de inflação mensal $(\dot{p})$, para avaliar se a variação da taxa cambial causaria aumento imediato da inflação; e

(e) spread do C-Bond $\left(Z_{t}\right)$ e taxa de câmbio nominal mensal $\left(E_{t}\right)$, para explicitar um dos possíveis canais que podem influenciar a taxa cambial.

O artigo está organizado em quatro seções. Após a introdução, na seção um, são apresentados os argumentos da visão hegemônica e os da perspectiva alternativa sobre as possibilidades da política monetária nas condições atuais, bem como alguns comentários sobre o debate em torno da hipótese Bresser-Nakano. Na seção dois são apresentados a metodologia e os resultados dos testes. Finalmente, na seção três, têm-se os comentários finais.

\section{INTEGRAÇÃO FINANCEIRA E TAXA DE JUROS}

Um dos assuntos mais debatidos nos meios acadêmicos e nos veículos de comunicação na atualidade tem sido, certamente, a questão da internacionalização das finanças, a globalização e suas potenciais influências sobre o ambiente doméstico das nações. A maior interconexão entre os mercados financeiros é marcada pelo grande avanço nas tecnologias de comunicação e informação, pela criação de um grande número de novos instrumentos financeiros pelos bancos e empresas, e, em especial, pelo processo de desregulamentação das economias, posto em marcha, no caso brasileiro, desde o início dos anos 1990.

Nesse ambiente, muitos economistas passaram a questionar a própria capacidade de um país manter uma taxa de juros doméstica em patamar compatível com um maior crescimento e emprego (Andrews, 1994a, 1994b). 
Isso porque, segundo esses autores, "when capital is highly mobile across international borders, the sustainable macroeconomic policy options available to states are systematically circumscribed" (Andrews, 1994b: 193-195). Em particular, a taxa básica de juros estaria menos sujeita à política discricionária do Banco Central e mais dependente da dinâmica financeira dos capitais internacionais e das políticas econômicas de outros países. ${ }^{6}$ A perda de autonomia da política monetária para definir a taxa de juros doméstica não estaria restrita ao regime de câmbio fixo, ${ }^{7}$ mas também ao regime de câmbio flutuante (Oreiro, 2002; Sicsú, 2002; Garcia, 2003).

Essa perspectiva está baseada em dois conceitos fundamentais que constituem a RIP (Rate Interest Parity), a qual assegura que ocorre a equalização dos juros entre os países em virtude dos fluxos de capitais (Frankel, 1992). Ela compreende duas relações que, a priori, devem ser equivalentes: a paridade coberta (CIP - Covered Interest Parity) e a paridade descoberta dos juros internacionais (UIP - Uncovered Interest Parity). A primeira assegura a equalização das taxas de juros entre os países em virtude dos fluxos de capitais, quando os ativos são contratados em uma moeda comum. Em geral, essa primeira proposição é geralmente aceita como empiricamente válida na literatura (Lavoie, 2000). ${ }^{8}$

Entretanto, com relação à UIP, muitos estudos empíricos têm-na rejeitado sistematicamente. ${ }^{9}$ A principal razão a que se aduz é que os agentes não são neutros ao risco. Ou seja, os títulos emitidos pelos governos dos diversos países não são substitutos perfeitos, em vista do risco de default, ${ }^{10}$ ou os agentes estariam antes interessados em diversificar seu portfólio (Lavoie, 2002-3). A hipótese da UIP ${ }^{11}$ assevera que a taxa de retorno entre os diversos ativos deve ser a mesma entre os países. Para o caso brasileiro, com relação aos Estados Unidos (EUA), por exemplo, ela pode ser expressa como:

$$
\frac{(R \$ / U S \$)^{e}}{(R \$ / U S \$)}=\frac{\left(1+i^{R \$}\right)}{\left(1+i^{U S \$}\right)}
$$

em que (R\$/US\$) é a taxa de câmbio spot esperada; ( $R \$$ / US\$) é a taxa de câmbio spot vigente; $i^{R \$}$ é a taxa de juros que remunera os títulos denominados em reais; e $i$ US\$ é a taxa de juros que remunera os títulos denominados em dólares. De (1) pode-se se obter: 


$$
\left(1+i^{R \$}\right)=(U S \$ / R \$) \cdot\left(1+i^{U S \$}\right)(R \$ / U S \$)^{e}
$$

Da perspectiva do Brasil, o lado esquerdo da equação (2) é a taxa de retorno que pode ser obtida domesticamente, e, do lado direito, o retorno que pode ser obtido quando se compram títulos denominados em dólares. Quando ocorre essa igualdade, o mercado de ativos está em equilíbrio. Se, porém, por algum motivo essa igualdade não se verifica, as forças do mercado restauram o equilíbrio.

Por exemplo, se o lado esquerdo de (2), por alguma razão, torna-se maior que o lado direito de (2), isso significa que os agentes obtêm uma taxa de retorno no Brasil mais alta que nos Estados Unidos. Em vista da perfeita mobilidade dos capitais e da neutralidade em relação ao risco, isso levaria a uma migração destes dos EUA para o Brasil, reduzindo $i^{R \$}$ e elevando $i^{U S \$}$, causando uma depreciação em termos de dólares (um aumento em US\$ I $R \$)$. Esse processo continuaria até que a igualdade da equação (1) fosse restabelecida.

De acordo com a hipótese da UIP, a taxa de juros doméstica só pode ser menor que a taxa internacional se os agentes têm a expectativa de uma apreciação da taxa de câmbio no futuro. Alternativamente, se os agentes esperam uma depreciação da taxa cambial, ela tem de ser compensada por uma taxa de juros doméstica superior à taxa internacional. Nessa perspectiva, Agénor e Montiel (2000) observam que, com perfeita mobilidade de capitais (entendida como sinônimo de substituição perfeita entre os ativos ${ }^{12}$ ), o ajuste da equação (2) é instantâneo, pois o mercado sempre ajusta seu portfólio produzindo a igualdade postulada pela paridade descoberta dos juros internacionais.

Desse modo, concluem que a fixação da taxa de juros doméstica pelos bancos centrais dos países em desenvolvimento, conforme a definição anterior, será inócua se não refletir aquela definida pelas forças do mercado, ajustada pelas expectativas. "El costo marginal de los fondos [de empréstimo] en la economía estaria dado entonces por la tasa de paridad no cubierta [UIP] y 'no se vería afectado por fenómenos internos' (...)" (Agénor e Montiel, 2000: 219; aspas acrescentadas).

No caso brasileiro, especificamente, a visão hegemônica é de que “(...) alto grau de abertura da conta de capitais (...) é um dos fatores que colocam 
a taxa nominal e real de juros do Brasil entre as mais altas do mundo" (Paula et al., 2003: 108; grifo do original). Isto é, a dinâmica financeira de compra e venda de títulos de dívida entre as nações estaria tornando o poder da autoridade monetária, para decidir sobre o nível da taxa básica de juros, mais aparente do que real. A causa básica seria a "perfeita mobilidade do capital" ou dos "fluxos de capitais". ${ }^{13}$ Nessas circunstâncias, as economias em desenvolvimento, como o Brasil, nada perderiam ao renunciar inteiramente à sua política monetária, pois a taxa de juros doméstica alcançaria o seu nível de equilíbrio sob a ação das forças de mercado através do mecanismo previsto na equação (2).

Para alguns economistas, porém, a taxa de juros doméstica é uma variável estritamente monetária, sujeita à manipulação política, mediante a qual o Banco Central exerce o controle sobre as demais variáveis do sistema (Kaldor, 1982; Moore, 1988; Lavoie, 1992). Está relacionada com a geração de uma discrepância ex post entre a taxa de câmbio futura $(R \$ / U S \$)_{t+1}$, negociada no período corrente sob contrato para entrega no período seguinte, $\mathrm{e}$ a taxa de câmbio que o mercado espera para o período seguinte $(R \$ / U S \$)^{e}$.

A autonomia da política monetária das pequenas economias abertas em ambiente de livre mobilidade de capitais e câmbio flexível, ${ }^{14}$ sumariada nos trabalhos de Paraskevopoulos et al. (1996); Paschakis e Smithin (1998); Smithin (1997, 1999, 2002-3, 2003, cap. 8); Kam e Smithin (2004); Lavoie (2000, 2002-3), está baseada no currency risk ou risk premium (Z). Se a CIP, por definição, sempre se mantém, o mesmo não ocorre com a UIP, pois esta requer necessariamente que os ativos sejam substitutos perfeitos (que os agentes sejam neutros em relação ao risco na composição de seus portfólios), o que difere de mobilidade perfeita de capitais [ausência da componente country risk (Frankel, 1992)]. ${ }^{15}$

Muitos estudos têm indicado que a perfeita substituição entre os ativos é uma hipótese pouco plausível nas condições contemporâneas. ${ }^{16}$ Evidências recentes sugerem que a hipótese da paridade descoberta dos juros internacionais (UIP) deve ser completamente rejeitada (Fujii e Chinn, 2001). ${ }^{17}$ Frankel (1992) argumenta que extensos diferenciais de juros entre as nações ainda persistem, pois os títulos emitidos pelos diversos governos são claramente substitutos imperfeitos. Esses diferenciais de juros expressam mais a autonomia da autoridade monetária na arena internacional do que a equalização das taxas de juros. Esse seria um dos motivos pelos quais “(...) even 
in open economy with financial mobility, central banks retain the ability to set interest rates of their choice, with a wide spectrum" (Lavoie, 2000: 163).

Nos países em desenvolvimento, onde a moeda está freqüentemente sob suspeita, a dívida externa líquida (como proporção do PIB) fornece um indicador para o observador estrangeiro de como a taxa de câmbio deve variar (Smithin, 2002-3). Essa situação, empregando-se a equação (2), pode ser expressa como:

$$
\left(1+i^{R \$}\right)=(U S \$ / R \$) \cdot\left(1+i^{U S \$}\right)(R \$ / U S \$)^{e}+Z,
$$

em que $Z$ decorre da percepção de risco dos agentes financeiros. Nessas circunstâncias, a UIP não necessariamente se mantém, pois algo como um currency risk ou credit risk deve existir, e a variável $Z$ é necessariamente diferente de zero. Por exemplo, se $Z>0$, os títulos brasileiros estão sob a suspeita dos agentes financeiros: o desequilíbrio da equação (1) se mantém indefinidamente; se $Z<0$, os agentes têm uma percepção de risco muito pequena e os títulos do Governo brasileiro exercem grande atratividade no mercado internacional: o Banco Central do Brasil define taxas de juros (exogenamente) tão baixas quanto os demais países em desenvolvimento e o desequilíbrio da equação (1), agora favorável, se mantém indefinidamente.

Desse modo, seria possível manter taxas de juros domésticas em níveis similares aos dos demais países em desenvolvimento em ambiente de perfeita mobilidade de capitais, manipulando unicamente a componente risk premium (Z) (Smithin, 1999). Uma especificação possível para a variável $Z$, segundo Smithin (1999, 2002-3) e Paraskevopoulos et al. (1996), é a seguinte:

$$
Z=z(D), Z_{D}^{\prime}>0
$$

em que $Z$ é uma função crescente da dívida externa líquida como proporção do PIB (D), ou, alternativamente, uma função crescente do spread do C-Bond. ${ }^{18} \mathrm{O}$ diferencial de juros, desse modo, deve variar consoante o risk premium da nação endividada. "We suggest that the risk premium should be related to some measure of the overall debt/credit position of the domestic economy" (Paraskevopoulos et al., 1996: 9).

Se na perspectiva hegemônica $Z=0$, em que o equilíbrio da equação (1) é sempre alcançado, na perspectiva alternativa ela pode ser tomada como uma constante positiva ou como a diferença residual entre as duas demais variáveis exogenamente determinadas, em que o "desequilíbrio" da equação (1) se 
mantém ad infinitum. Seguindo Smithin (2002-3) e Cirne de Toledo (2002), essa componente pode ser reduzida simplesmente a um spread entre as duas demais taxas exógenas. Em notação matemática, tem-se:

$$
Z=f\left(i^{R \$}-i^{U S \$}\right)
$$

Portanto, quaisquer discrepâncias de curto e longo prazo entre as taxas de juros do Brasil e do exterior serão sempre acomodadas por variações na posição credora (devedora) do país no mercado internacional. Alternativamente, isso deve refletir uma melhora (piora) do status dos títulos da dívida pública do país em consideração. Na perspectiva alternativa, um país faz surgir a componente risk premium $(Z)$ na medida em que decide manter sistematicamente uma taxa de juros doméstica maior que a taxa internacional (Lavoie, 2000: 169).

A seguir, os testes de causalidade visam a captar o sentido da causalidade e o sinal da correlação entre a taxa de juros e o risco-país para a economia brasileira. E, em um segundo momento, identificar qual seria o impacto de uma redução progressiva da taxa de juros doméstica sobre as demais variáveis a ela vinculadas, como a taxa cambial e a taxa de inflação.

\section{ANÁLISE EMPÍRICA PARA A ECONOMIA BRASILEIRA}

\subsection{Modelo teórico}

O teste de causalidade de Granger $(1969,1988)$ e Granger et al. (1998) assegura que, à medida que se obtêm melhores predições para uma dada série $y$, adicionando-se valores defasados de uma dada série $x$, diz-se que $x$ está causando $y$ no sentido de Granger, isto é, existe uma relação líder-defasagem entre $x$ e $y$. Neste caso, $x$ exerce controle sobre $y$ (Granger, 1988). Os demais resultados possíveis do teste são tais que: $y$, contrariamente, esteja causando $x$; não haja nenhuma relação de dependência entre as variáveis; ou haja mútua dependência entre ambas (feedback).

A seqüência do teste de causalidade requer, primeiramente, a investigação da existência de raízes unitárias e co-integração entre as variáveis analisadas. Os resultados desses testes encontram-se nas tabelas 3 a 8 . Se as séries $x$ e $y$ são co-integradas, a causalidade entre as variáveis é investigada mediante o seguinte modelo (Granger et al., 1998: 5): 


$$
\begin{aligned}
& \Delta y_{t}=\alpha_{0}+\delta_{1} \hat{\varepsilon}_{t-1}+\sum_{i=1}^{k} \alpha_{1 l} \Delta y_{t-i}+\sum_{i=1}^{k} \alpha_{2 i} \Delta x_{t-i}+v_{1 t} \\
& \Delta x_{l}=\beta_{0}+\delta_{2} \hat{\varepsilon}_{t-1}+\sum_{i=1}^{k} \beta_{1 i} \Delta y_{t-i}+\sum_{i=1}^{k} \beta_{2 i} \Delta x_{t-i}+v_{2 t}
\end{aligned}
$$

em que $\hat{\varepsilon}_{t-1}$ é o termo de correção de erro proveniente da regressão co-integrante, e $\hat{\delta}_{1}$ e $\hat{\delta}_{2}$ são as velocidades de ajustamento das séries.

Se, porém, $x$ e $y$ não são co-integradas, em que $\hat{\varepsilon}_{t}$ é $I$ (1), a causalidade entre as séries pode ser investigada mediante o seguinte modelo (Granger et al., 1998: 5):

$$
\begin{aligned}
& \Delta y_{t}=\alpha_{0}+\sum_{i=1}^{k} \alpha_{1 i} \Delta y_{t-i}+\sum_{i=1}^{k} \alpha_{2 i} \Delta x_{t-i}+\mu_{1 t} \\
& \Delta x_{t}=\beta_{0}+\sum_{i=1}^{k} \beta_{1 i} \Delta y_{t-i}+\sum_{i=1}^{k} \beta_{2 i} \Delta x_{t-i}+\mu_{2 t}
\end{aligned}
$$

Alternativamente, se ambas as séries $x$ e $y$ são estacionárias [I(0)], a causalidade entre ambas é investigada estimando-se o seguinte modelo (Granger, 1969: 431):

$$
\begin{aligned}
& y_{t}=\alpha_{0}+\sum_{i=1}^{k} \alpha_{1 i} y_{t-i}+\sum_{i=1}^{k} \alpha_{2 i} x_{t-i}+\eta_{1 t} \\
& x_{t}=\beta_{0}+\sum_{i=1}^{k} \beta_{1 i} y_{t-i}+\sum_{i=1}^{k} \beta_{2 i} x_{t-i}+\eta_{2 t}
\end{aligned}
$$

Considerando-se (6), (7) e (8), se não for possível rejeitar $H_{0}: \alpha_{21}=\alpha_{22 \ldots}$ $=\alpha_{2 k}=0$ e simultaneamente $\delta_{1}=0$, a variável $x$ não causa $y$ no sentido de Granger; alternativamente, caso não seja possível rejeitar $H_{0}: \beta_{11}=\beta_{12 \ldots}=\beta_{1 k}$ $=0$ e simultaneamente $\delta_{2}=0$, a variável $y$ não causa $x$ no sentido de Granger. Nos testes a seguir a extensão das defasagens obedeceu ao Critério de Informação de Schwartz (minimização), e, para reforçar as conclusões dos testes ADF, foram incluídas as saídas do teste tradicional de Philips-Perron (PP), sugerido pela literatura corrente (Patterson, 2000). 


\subsection{Resultados ${ }^{19}$}

O resumo dos testes de causalidade a partir dos modelos (6), (7) e (8) aparece na tabela 2 , de onde se pode concluir sobre o sentido das causalidades entre as variáveis em consideração e o sinal de sua correlação. As estimativas de cada regressão co-integrante estão listadas no apêndice ( $a$ até $f$ ).

\subsection{Discussão dos resultados}

Os resultados dos testes de causalidade de Granger, expostos na tabela 2, permitem que se façam as seguintes afirmações para o período analisado:

(1) a taxa de juros Selic mensal $\left(i_{t}\right)$ precede o spread do C-Bond $\left(Z_{t}\right)$ e tem correlação positiva com este, indicando que uma elevação da taxa de juros pelo Banco Central eleva a percepção de risco dos agentes financeiros internacionais;

(2) a taxa de juros Selic $\left(i_{t}\right)$ precede a taxa de câmbio diária $\left(E_{t}\right)$ e exibe uma correlação positiva com esta, indicando que a elevação dos juros pela autoridade monetária tende a depreciar a taxa de câmbio no Brasil;

(3) a taxa de juros Selic $\left(i_{t}\right)$ e a variação do IPCA $\left(\dot{p}_{t}\right)$, como em outros trabalhos ${ }^{20}$ são estatisticamente independentes, indicando que a taxa de juros não exerce influência direta sobre a taxa de inflação;

(4) a variabilidade da taxa de juros Selic $\left(\dot{i}_{t}\right)$ e a variabilidade da taxa cambial $\left(\dot{E}_{t}\right)$ são estatisticamente independentes, indicando que a variabilidade da taxa de juros não exerce influência sobre a volatilidade cambial;

(5) a variabilidade da taxa cambial $\left(\dot{E}_{t}\right)$ precede a inflação mensal $\left(\dot{p}_{t}\right)$, conquanto não se possa afirmar que essa variabilidade seja transmitida diretamente para o IPCA, pois o parâmetro co-integrante é largamente não significativo; ${ }^{21} \mathrm{e}$

(6) o spread do C-Bond $\left(Z_{t}\right)$ precede e tem correlação positiva com a taxa cambial $\left(E_{t}\right)$, indicando que a variável risk premium exerce influência positiva sobre a taxa cambial, reforçando o impacto dos juros no sentido da depreciação. Desses resultados seguem as seguintes considerações.

Primeiramente, dos resultados mostrados se pode inferir que uma elevação da taxa de juros Selic por parte da autoridade monetária, qualquer que seja o motivo, tende a elevar a percepção de risco dos agentes financei- 
ros internacionais, e não o contrário. Esse resultado apóia a hipótese Bresser-Nakano.

Outro aspecto importante é que, para o período em análise, com dados diários, a hipótese aventada por Arida (2002), de que elevações na taxa de juros Selic poderiam levar a uma depreciação da taxa de câmbio no Brasil, é também apoiada por esses resultados. Portanto, uma redução na taxa de juros doméstica tenderia a apreciar a taxa cambial, e não o contrário. Esse achado, portanto, não apóia o argumento de Oreiro (2002), para quem a redução da taxa de juros causaria depreciação cambial.

Segundo Arida, a explicação para tanto é de que, "no caso anômalo, o efeito default domina o efeito juros" (Arida, 2002: 126). Haveria uma "curva BB” para o Brasil, em que, segundo o autor, no seu segmento normal, “(...) aumentos na taxa de juros valorizam a moeda nacional; [porém,] no seu segmento anômalo geram uma percepção de risco tão negativa que terminam por depreciar a moeda nacional" (Arida, 2002: 126). Conforme os resultados anteriores, esse parece ser o caso mais representativo para a economia brasileira para o período analisado.

Outro resultado marcante é que a taxa de juros não exerce, estatisticamente, influência sobre a taxa de inflação (IPCA), nem esta sobre a taxa de juros. $^{22}$ Teoricamente, seria esperada uma relação inversa ex ante entre essas variáveis, como indicam os modelos de metas de inflação (Bogdanski et al., 2000). De acordo com os resultados obtidos, o Banco Central, ao elevar a taxa de juros, não produz os resultados que o modelo de metas prevê. A não ser que essa influência não possa ser captada estatisticamente por essa metodologia. Este é, possivelmente, um dos motivos pelos quais se diz que a política de juros altos é ineficaz contra a inflação inercial, visto que não afeta a tendência inflacionária (Lopes, 1985). ${ }^{23}$

Com relação à variação da taxa de juros e à sua relação com a variação diária da taxa de câmbio, os testes indicam que ocorre independência entre ambas. A variação da taxa de juros não exerce influência sobre a variação da taxa de câmbio (apêndice $d$ ). Esses resultados não endossam, portanto, afirmações como as de Sicsú (2002), de que o Banco Central não tem autonomia para reduzir a taxa de juros, haja vista a variação da taxa de câmbio que ela engendraria. Simetricamente, os resultados dos testes apontam para a efetiva possibilidade de o Banco Central fixar a taxa de juros em um nível 
mais baixo sem elevar a volatilidade da taxa cambial, em vista da independência entre essas variáveis.

Outro resultado que, a priori, seria esperado é de que a variabilidade cambial preceda a taxa de inflação mensal: haveria um pass-through do câmbio para a taxa de inflação. ${ }^{24} \mathrm{~A}$ precedência vai da variabilidade do câmbio para a taxa de inflação, a 5\% de significância para o curto período analisado. É importante reter, porém, como visto, que a estimativa da equação co-integrante e seus parâmetros são largamente não significativos. Isso indica que a influência da variação cambial não é exercida diretamente sobre a taxa de inflação, como supõe o argumento de Oreiro (2002). Esses canais de transmissão permanecem ainda em aberto.

Sobre a precedência do risco e sua correlação positiva com a taxa cambial, é preciso destacar que este é um resultado previsto pelo modelo do Banco Central (Bogdanski et al., 2000). O que fica mais claro a partir dos resultados mostrados na tabela 2, porém, é que aumentos da taxa de juros elevam o spread do C-Bond e também a taxa cambial, ou seja, a taxa cambial recebe um duplo impacto no sentido da depreciação. Este pode ser um dos possíveis canais pelos quais, em um ambiente com inércia inflacionária, o combate à inflação pela elevação da taxa de juros não produza os resultados esperados. Na expressão de Arida, o "efeito default domina o efeito juros". É o estigma de um caso anômalo da política monetária. ${ }^{25}$

É preciso ressaltar, a partir dos resultados anteriores, que a possibilidade de migração para um novo regime de taxa de juros, segundo a proposta de Bresser e Nakano, baseia-se na persistência de uma grande parcela da dívida pública indexada à taxa Selic e, provavelmente, em uma conjuntura externa também favorável. ${ }^{26}$ Isso porque a redução da taxa básica afetaria diretamente o endividamento público, reduzindo-o, bem como a percepção de risco dos agentes financeiros. A simetria postulada neste trabalho apóia-se nessa premissa básica.

Nesse aspecto, é de se esperar que ela de fato se verifique, uma vez que a parcela da dívida pública indexada à taxa Selic, nesse período, permaneceu a mesma, ${ }^{27}$ havendo, por outro lado, uma substituição entre a parcela da dívida indexada ao câmbio, atualmente nula, e a parcela da dívida indexada ao IPCA, que aumentou. ${ }^{28}$ Posto que o IPCA está atualmente estabilizado em patamares internacionais ( $3-4 \%$ ao ano), isso tudo indica uma liberdade ainda maior para a autoridade monetária migrar para um regime de taxa de 
juros em patamares internacionais, uma vez que a taxa cambial pode variar mais livremente (para cima, espera-se), em uma velocidade e um espectro bem maiores do que antes, posto que agora a dívida pública é independente dessa variável. Nessas condições, é bastante razoável esperar que a política de redução progressiva da taxa básica de juros proposta por Bresser e Nakano, se fosse implementada pela autoridade monetária, provocasse os resultados previstos em seu argumento.

\section{COMENTÁRIOS FINAIS}

O objetivo principal deste estudo foi investigar a validade empírica da hipótese central de Bresser e Nakano (2002) para a economia brasileira. Segundo esses autores, seria factível que o Banco Central do Brasil adotasse uma política de redução progressiva da taxa de juros por ele estabelecida. O fundamento básico de seu argumento é de que a taxa de juros tem precedência e estaria positivamente correlacionada com o risco-país. Os resultados deste estudo são expressivos e apóiam a hipótese anteriormente delineada: para os últimos 10 anos de política monetária no Brasil, aumentos na taxa de juros pelo Banco Central, qualquer que seja a razão, tendem a elevar a percepção de risco dos agentes financeiros, e não o contrário. A causalidade vai dos juros estabelecidos pela autoridade monetária para o prêmio de risco, e não o inverso.

Assim, uma política de redução progressiva da taxa de juros, nessas condições, seria factível: ao fazê-lo, a autoridade monetária reduz a percepção de risco do mercado e eleva a taxa de crescimento da economia, melhorando, concomitantemente, a solvência das contas públicas ${ }^{29}$ sem comprometer a estabilidade de preços. A redução da taxa de juros poderia apreciar a taxa cambial por dois distintos canais: a taxa de juros tem precedência e uma correlação direta com a taxa cambial; depois, o menor risco-país, em vista da redução na taxa de juros, também precede e tem relação direta com a taxa cambial. Ao final, portanto, dependendo dos parâmetros, poder-se-ia especular até mesmo uma redução da taxa de inflação ${ }^{30}$ em vista da apreciação da taxa cambial conjugada com maior crescimento econômico.

Enfim, os resultados são coerentes com os de outros estudos recentes, como o de Gonçalves e Guimarães (2005), e sugerem que seria viável para o Brasil, nas condições atuais, alcançar um regime de taxa de juros em níveis 
similares aos dos demais países em desenvolvimento (conforme a tabela 1), como sugeriram Bresser e Nakano (2002), possibilitando, com isso, um círculo virtuoso de crescimento e emprego sem comprometer a estabilidade, melhorando a credibilidade e os fundamentos da economia.

\section{APÊNDICE}

Quadro 1: Estimativas das regressões co-integrantes ${ }^{\Psi}$

\begin{tabular}{ll}
\hline \multicolumn{1}{c}{$a$} & \multicolumn{1}{c}{$b$} \\
\hline$\hat{Z}=217,11+3,24 t+223,25 i$ & $\hat{E}=0,67+0,001 t+0,0061 i$ \\
$\mathrm{~V} \cdot \mathrm{p}(0,034)(0,002)(0,000)$ & $\mathrm{V} \cdot \mathrm{p}(0,000)(0,000)(0,000)$ \\
$\mathrm{R}^{2}=0,1472 ;$ & $\mathrm{R}^{2}=0,7323 ;$ \\
$\mathrm{F}(1 ; 120)=10,27(0,000)$. & $\mathrm{F}(1 ; 1359)=1855,93(0,000)$. \\
\hline \multicolumn{1}{c}{$c$} & \multicolumn{1}{c}{$d$} \\
\hline \multicolumn{1}{c}{$e$} & $\hat{\dot{E}}=0,034-0,024 i$ \\
$\hat{\dot{E}}=0,70-0,018 \dot{p}$ & $\mathrm{~V} \cdot \mathrm{p}(0,189)(0,032)$ \\
$\mathrm{V} \cdot \mathrm{p}(0,000)(0,351)$ & $\mathrm{R}=0,0006 ;$ \\
$\mathrm{R}^{2}=0,8722 ;$ & $\mathrm{F}(1 ; 1359)=0,768(0,381)$. \\
$\mathrm{F}(1 ; 66)=1,40(0,241)$. & \\
\hline & \\
\hline$\hat{\dot{p}}=-0.369-0,001 t+0,71 i$ & $\hat{E}=-0,025+0,022 t+0,00072 Z$ \\
$\mathrm{~V} \cdot \mathrm{p}(0,491)(0,584)(0,058)$ & $\mathrm{V} \cdot \mathrm{p}(0,676)(0,000)(0,000)$ \\
$\mathrm{R}^{2}=0,0919 ;$ & $\mathrm{R}=0,9493 ;$ \\
$\mathrm{F}(1 ; 66)=3,24(0,046)$. & $\mathrm{F}(1 ; 121)=1114,87(0,000)$. \\
\hline
\end{tabular}

世 P-valor entre parênteses. Os sinais da correlação entre as variáveis, expostos na tabela 7, são simplesmente os sinais dos parâmetros das variáveis explicativas das equações acima.

Tabela 1: Taxas juros praticadas no mundo - países emergentes

\begin{tabular}{llll}
\hline País & Taxa de juros nominal (\% a.a.) & \multicolumn{1}{c}{ País } & Taxa de juros nominal (\% a.a.) \\
\hline China & 2,20 & Peru & 3,01 \\
\hline Hong Kong & 2,29 & Venezuela & 12,70 \\
\hline Índia & 2,31 & África do Sul & 7,10 \\
\hline Indonésia & 7,72 & República Checa & 2,03 \\
\hline Malásia & 2,82 & Hungria & 7,69 \\
\hline Filipinas & 6,00 & Polônia & 5,50 \\
\hline Cingapura & 2,13 & Rússia & 13,00 \\
\hline Coréia do Sul & 3,51 & Tailândia & 2,65 \\
\hline Taiwan & 1,40 & Argentina & 4,63 \\
\hline Colômbia & 7,25 & Brasil & 19,24 \\
\hline México & 9,66 & Chile & 3,48 \\
\hline
\end{tabular}

Fonte: The Economist, p. 100, 23.4.2005. 
Tabela 2: Resultados dos testes de causalidade

\begin{tabular}{cccc}
\hline Sentido da causalidade & Valor-P (Teste Wald) & Estatística F & Correlação* $^{*}$ \\
\hline$i_{t} \Rightarrow Z_{t}$ & 0,000 & 11,24 & + \\
\hline$Z_{t} \Re i_{t}$ & 0,122 & 2,14 & $?$ \\
\hline$i_{t} \Rightarrow E_{t}^{\#}$ & 0,034 & 2,61 & $?$ \\
\hline$E_{t} \Re i_{t}$ & 0,703 & 0,59 & $?$ \\
\hline$i_{t} \Re \dot{p}_{t}$ & 0,663 & 0,41 & $?$ \\
\hline$\dot{p}_{t} \Re i_{t}$ & 0,279 & 1,31 & $?$ \\
\hline$\dot{i}_{t} \Re \dot{E}_{t}$ & 0,710 & 0,34 & $?$ \\
\hline$\dot{E}_{t} \Re \dot{i}_{t}$ & 0,419 & 0,87 & $?$ \\
\hline$\dot{E}_{t} \Rightarrow \dot{p}_{t}^{\# \#}$ & 0,027 & 5,13 & $?$ \\
\hline$\dot{p}_{t} \Re \dot{E}_{t}$ & 0,281 & 1,18 & + \\
\hline$Z_{t} \Rightarrow E_{t}$ & 0,002 & 10,22 & $?$ \\
\hline$E_{t} \Re Z_{t}$ & 0,106 & 2,65 & $?$ \\
\hline
\end{tabular}

Fonte: Cálculos dos autores a partir de dados do IPEADATA. \# a 5\% de significância. *Sinal do parâmetro co-integrante, conforme Apêndice ( $a$ até $f$ ). \#\# A estimativa da equação co-integrante é largamente não significativa. Ver apêndice $c$. Legenda: $\Rightarrow$ indica causalidade unidirecional; $\Re$ indica independência; $\Leftrightarrow$ indica dependência mútua (feedback).

Tabela 3: Teste de raiz unitária e co-integração para a taxa de juros Selic mensal e o spread do C-Bond em pontos-base mensal, 1995:01 a 2005:02

\begin{tabular}{ccccc}
\hline Variáveis & $\mathrm{ADF}{ }^{\star \S}$ & Valor-P (ADF) & $\mathrm{PP}$ $\S$ & Valor-P (PP) \\
\hline$i_{t}$ & $-2,95$ & 0,151 & $-2,97$ & 0,145 \\
\hline$Z_{t}$ & $-2,78$ & 0,208 & $-2,33$ & 0,410 \\
\hline$\Delta i_{t}$ & $-11,26$ & 0,000 & $-11,35$ & 0,000 \\
\hline$\Delta Z_{t}$ & $-7,18$ & 0,000 & $-7,15$ & 0,000 \\
\hline$\hat{\varepsilon}_{t}$ & $-2,91^{* *}$ & \multicolumn{3}{c}{ Não se rejeita a hipótese nula } \\
\hline
\end{tabular}

*Valores tabelados: ADF = PP 1\% (-4,04); ADF = PP 5\% $(-3,45)$.

* * Os valores críticos para a estimativa dos erros da regressão co-integrante foram calculados conforme a tabela A8.1

(Patterson, 2000: 372): C $(1 \%, 122)=-4,8225 ; C(5 \%, 122)=4,2187$. § Com constante e tendência.

Tabela 4: Teste de raiz unitária e co-integração para a taxa de juros Selic diária e taxa de câmbio nominal diária, 2.8.1999 a 6.1.2005

\begin{tabular}{ccccc}
\hline Variáveis & ADF* & Valor-P (ADF) & PP* & Valor-P (PP) \\
\hline$i_{t}$ & $-1,68$ & 0,443 & $-1,15$ & 0,696 \\
\hline$E_{t}$ & $-1,41$ & 0,579 & $-1,44$ & 0,561 \\
\hline$\Delta i_{t}$ & $-5,50$ & 0,000 & $-35,91$ & 0,000 \\
\hline$\Delta E_{t}$ & $-27,18$ & 0,000 & $-30,33$ & 0,000 \\
\hline$\hat{\varepsilon}_{t}$ & $-1,68^{* *}$ & & Não se rejeita a hipótese nula & \\
\hline
\end{tabular}

*Valores tabelados: ADF = PP 1\% (-3,43); ADF = PP 5\% (-2,86);

* * Os valores críticos para a estimativa dos erros da regressão co-integrante foram calculados conforme a tabela A8.1 (Patterson, 2000: 372): C (1\%, 1360) $=-4,6812 ; C(5 \%, 1360)=-4,1281$. 
Tabela 5: Teste de raiz unitária e co-integração para a taxa de juros Selic mensal e taxa de inflação mensal, 1999:07 a 2005:01

\begin{tabular}{ccccc}
\hline Variáveis & ADF*§ & Valor-P (ADF) & PP*§ & Valor-P (PP) \\
\hline$\dot{P}_{t}$ & $-4,03$ & 0,012 & $-4,12$ & 0,009 \\
\hline$i_{t}$ & $-2,26$ & 0,448 & $-3,12$ & 0,109 \\
\hline$\Delta \dot{P}_{t}$ & $-8,07$ & 0,000 & $-13,03$ & 0,000 \\
\hline$\Delta i_{t}$ & $-3,77$ & 0,0247 & $-12,12$ & 0,000 \\
\hline$\hat{\varepsilon}_{t}$ & $-4,24^{* *}$ & Não se rejeita a hipótese nula \\
\hline
\end{tabular}

*Valores tabelados: ADF = PP $1 \%(-4,10) ;$ ADF = PP $5 \%(-3,48)$.

** Os valores críticos para a estimativa dos erros da regressão co-integrante foram calculados conforme a tabela A8.1 (Patterson, 2000: 372): $C(1 \%, 67)=-4,9546 ; C(5 \%, 67)=-4,3017$. § Com constante e tendência.

Tabela 6: Teste de raiz unitária para a variabilidade da taxa de juros Selic diária e variabilidade da taxa de câmbio nominal diária, 2.8.1999 a 6.1.2005

\begin{tabular}{ccccc}
\hline Variáveis & $\mathrm{ADF} * \S$ & Valor-P (ADF) & $\mathrm{PP} * \S$ & Valor-P (PP) \\
\hline$\dot{i}_{t}$ & $-35,75$ & 0,000 & $-35,74$ & 0,000 \\
\hline$\dot{E}_{t}$ & $-27,45$ & 0,000 & $-31,07$ & 0,000 \\
\hline
\end{tabular}

*Valores tabelados: ADF = PP 1\% $(-3,43) ;$ ADF $=$ PP 5\% $(-2,86)$. § Com constante.

Tabela 7: Teste de raiz unitária para a taxa de inflação mensal e a variação percentual da taxa de câmbio nominal mensal, 1999:07 a 2005:01

\begin{tabular}{|c|c|c|c|c|}
\hline Variáveis & $A D F * \S$ & Valor-P (ADF) & $\mathrm{PP} * \S$ & Valor-P (PP) \\
\hline$\dot{E}_{t}$ & $-5,32$ & 0,000 & $-5,31$ & 0,000 \\
\hline$\dot{P}_{t}$ & $-4,03$ & 0,002 & $-4,13$ & 0,002 \\
\hline
\end{tabular}

*Valores tabelados: ADF = PP 1\% $(-3,54) ;$ ADF = PP 5\% $(-2,91) . \S$ Com constante

Tabela 8: Teste de raiz unitária e co-integração para a taxa câmbio nominal mensal e spread do C-Bond em pontos base mensal, 1995:01 a 2005:02

\begin{tabular}{ccccc}
\hline Variáveis & ADF* & Valor-P (ADF) & PP* & Valor-P (PP) \\
\hline$Z_{t}$ & $-2,78$ & 0,208 & $-2,33$ & 0,410 \\
\hline$E_{t}$ & $-2,24$ & 0,460 & $-2,16$ & 0,508 \\
\hline$\Delta Z_{t}$ & $-7,18$ & 0,000 & $-7,15$ & 0,000 \\
\hline$\Delta E_{t}$ & $-7,75$ & 0,000 & $-7,699$ & 0,000 \\
\hline$\hat{\varepsilon}_{t}$ & $-2,97^{* *}$ & \multicolumn{3}{c}{ Não se rejeita a hipótese nula } \\
\hline
\end{tabular}

*Valores tabelados: ADF = PP 1\% $(-4,04) ;$ ADF = PP 5\% $(-3,44)$;

* * Os valores críticos para a estimativa dos erros da regressão co-integrante foram calculados conforme a tabela A8.1

(Patterson, 2000: 372): C (1\%, 122) $=-4,8225 ; C(5 \%, 122)=-4,2187$ 


\section{NOTAS}

1. Os dados disponibilizados pelo Banco Central do Brasil indicam que, para o período que vai de janeiro de 2001 a fevereiro de 2005, em média, 45,48\% do estoque de dívida pública estavam indexados à taxa de juros Selic. Para o período que vai de março de 2005 a março de 2007, essa média cresceu para 48,31\%.

2. "The essential features of the horizontalist approach to monetary economics are that credit and money are demand-led endogenous variables, and that central banks have the ability to set interest rates (even real interest rates), at a level of their choice (...). In a modern monetary production economy, there is no such thing as a natural rate of interest, towards which the central bank would necessarily have to align its bank rate. The same features still characterize an open economy operating in world where capital is mobile. Through the compensation principle, which is a variant of the reflux principle, balance of payments disequilibria have no effect on the overall monetary base or money supply, even with fixed exchange rates" (Lavoie, 2001: 237). No Brasil, a posição predominante tem sido a de que a integração financeira e a livre mobilidade dos capitais levam à perda de autonomia da política monetária. Essa é uma das razões pelas quais propõem a imposição de controles sobre a livre mobilidade dos capitais. Para uma discussão mais extensa ver, por exemplo, Ferrari Filho et al. (2003); Paula et al. (2003); Sicsú et al. (2003); Sicsú (2002); Bresser e Nakano (2002); Oreiro (2002).

3. A redução da taxa de juros pela autoridade monetária pode reduzir (duplamente) a razão dívida/PIB do País em virtude da redução líquida nos serviços da dívida (redução do numerador), e também aumentar a taxa de crescimento do PIB. O gasto com juros em 2004 correspondeu a quatro vezes o deficit do INSS: R\$ 128 bilhões. Embora o resultado primário seja expressivo, o resultado nominal ainda continua negativo, em vista dos expressivos gastos com juros elevados. Em média, cerca de $23 \%$ da carga tributária no Brasil são despendidos com os juros estabelecidos pelo Banco Central.

4. Ver, por exemplo, para o caso brasileiro, os comentários relativos ao artigo de Bresser e Nakano (2002) feitos por Oreiro (2002); Sicsú (2002); Arida (2002); e Cirne de Toledo (2002). Ver, também, as avaliações da política econômica atual e as alternativas sugeridas na coletânea Agenda Brasil: políticas econômicas para o crescimento com estabilidade de preços, em Sicsú et al. (2003).

5. Seguiu-se a metodologia de Granger (1969, 1988); Granger et al. (1998); Engle e Granger (1987); e Patterson (2000).

6. Sicsú, por exemplo, assegura que, nas condições contemporâneas: "Não existe liberdade (discrição) para o Banco Central manipular a taxa de juros (...)” (Sicsú, 2002: 134).

7. Seguindo os ensinamentos do Modelo Mundell-Fleming.

8. Para pós-keynesianos, a razão pela qual essa condição é sempre empiricamente satisfeita resulta da aplicação, pelos bancos comerciais, de uma regra de markup ao negociarem vendas de divisas. Para mais detalhes, ver Lavoie (2000) e especialmente Coulbois e Prissert (1974). 
9. Para a rejeição dessa hipótese no caso brasileiro, pode-se consultar Holanda e Cavalcante (2001). Vale notar que as provas empíricas para a rejeição da UIP foram apresentadas recentemente em Fujii e Chinn (2001) para um grande número de países.

10. Na verdade, o risco de default é um dos elementos componentes da variável risk premium, seguindo a definição empregada por Frankel (1992).

11. O modelo de metas de inflação do Banco Central do Brasil adota essa suposição. Para conhecer o modelo de metas do Banco Central, seus postulados e equações fundamentais, pode-se consultar Bogdanski et al. (2000).

12. Para os autores, a ausência de barreiras nas fronteiras nacionais (livre mobilidade de capitais) é condição suficiente para tornar os agentes neutros ao risco. Alguns autores, como Smithin (2002-3) e Lavoie (2000, 2002-3), porém, observam que a ausência de barreiras à mobilidade de capitais não tem necessariamente ligação direta com a função preferência dos agentes e seu comportamento relativo ao risco. Segundo Lavoie: "Capital may be perfectly mobile from one country to the next, no restrictions impeding the movements of capital from one country to another, without asset holders considering that all assets are perfect substitutes. Indeed, even within a closed economy, the better-known models of asset choice by households or financial corporations assume away perfect substitutability on the basis of portfolio choice theory" (Lavoie, 2002-3: 238). Nessa direção, outros estudos concluem que "(...) choices involving gains are usually risk averse, and choices involving losses are often risk seeking” (Tversky e Kahneman, 1990: 64).

13. É notável que esse tipo de argumento tenha também comportamento cíclico. Ver Branson (1970).

14. A condição necessária para a autonomia da política monetária de uma pequena economia aberta é a existência de diferentes sistemas monetários entre as economias nacionais, e que a taxa cambial seja potencialmente livre para flutuar (ainda que não se espere nenhuma variação). Em uma União Monetária (como a Zona do Euro), os países perdem inteiramente sua autonomia para implementar políticas monetárias (Ferrari Filho, 2001-2: 240; Smithin, 2002-3: 221).

15. Como observa Smithin, "the international investors must be indifferent as to precisely which 'promises to pay' and in which proportions the investors holds at any given moment a portfolio (U. S. dollars, Canadian dollars, Mexican pesos, euros, or yen). This seems unlikely, on the face of it, as long as there are separate currencies 'backed' by sovereign governments, and exchange rates are liable to change" (Smithin, 2002-3: 226).

16. "The absence of an exchange risk premium constitutes 'perfect capital substitutability'. This condition arises when government bonds, denominated in differing currencies, are treated as perfect substitutes. Investors will act this either when they are risk neutral (...)" (Fujii e Chinn, 2001: 5). No caso brasileiro, em seu modelo de metas de inflação, o BC adota a suposição de que a UIP é ajustada ex post pelo prêmio de risco (Z) (Ver Bogdanski et al., 2000). Isto é, cada vez que equação (2) não se verifica, ex post, é sempre possível afirmar que era precisamente essa a percepção (exógena) de risco do mercado, independentemente da taxa de juros fixada previamente e de seu impacto sobre as contas públicas (razão Dívida/PIB). 
17. "As in numerous previous studies (...) the real interest parity (RIP) hypothesis is decisively rejected whit short horizon data" (Fujii e Chinn, 2001:2).

18. O spread do C-Bond, em pontos base, aumenta fortemente com a suspeita dos agentes internacionais sobre a capacidade de o país, sob regime de câmbio flutuante, "honrar" seus compromissos no mercado de títulos, sem depreciar sua moeda. Essa variável é representativa do status da variável $Z$ para a economia brasileira nas condições atuais. Como observam Rocha e Moreira (2003), essa variável tem implicações decisivas sobre o crescimento econômico do País e sobre o custo de rolagem da dívida pública. Medido em pontos-base, esse spread expressa o diferencial entre a taxa de juros paga pelo Governo brasileiro e a taxa de juros paga pelo Governo norte-americano para um título de igual maturidade: 1.000 pontos-base equivalem, por exemplo, a um diferencial de $10 \%$ ao ano. Essa é a variável que o BC considera empiricamente relevante em seu modelo (Bogdanski et al., 2000).

19. Todas as estimativas foram realizadas empregando-se o software econométrico E-views 4.1.

20. Ver Cardoso e Vieira (2004), por exemplo.

21. A estimativa dessa equação é não significativa. Ver Apêndice $\boldsymbol{c}$.

22. Para outro estudo com o mesmo resultado, ver Cardoso e Vieira (2004).

23. O caráter marcadamente inercial da inflação brasileira vem sendo constatado em estudos recentes que apóiam a sua presença nos últimos 12 anos (Fasolo e Portugal, 2003; Figueiredo e Ferreira, 2002). Fasolo e Portugal constataram: 1) “(...) high persistence (inertia) of Brazilian inflation (...)". 2) "inflation does have an autonomous inertial component, without linkage to shocks in individual markets” (Fasolo e Portugal, 2003: 1).

24. Para um estudo recente sobre o assunto, ver Cardoso e Vieira (2004).

25. Outros estudos vêm encontrando uma correlação negativa entre as reservas e a taxa de juros no Brasil (Silva Jr. e Silva, 2004).

26. A manutenção de um saldo positivo na balança comercial, por exemplo. Na realidade, a migração gradual para um novo regime de taxa de juros tenderia a reforçar esse panorama, uma vez que atuaria como uma redução nos custos monetários de produção das empresas. Nesse novo regime, a relação inversa entre taxa cambial e taxa de juros voltaria a ser obtida, reforçando a possibilidade de uma balança comercial favorável.

27. Ver nota 1.

28. Em fevereiro de 2005 essa parcela era de 2,7\%.

29. A solvência das contas públicas proporciona uma maior atratividade para os títulos do País no mercado financeiro internacional. Atualmente cerca de um terço da carga tributária brasileira é despendida com juros pelo Governo, em vista da política de juros do Banco Central.

30. A redução na taxa de juros pode ser interpretada como um choque positivo de oferta (Smithin, 2001). 


\section{REFERÊNCIAS BIBLIOGRÁFICAS}

ANDREWS, D. (1994a) "Capital mobility and monetary adjustment in Western Europe, 19731991”, Policy Sciences, n. 27, p. 425-445.

(1994b) "Capital mobility and state autonomy: toward a structural theory of international monetary relations", International Studies Quarterly, v. 38, n. 2, p. 193-218.

AGÉNOR, P. R., MONTIEL, P. J. (2000) La Macroeconomía Del Desarrollo. México: Fundo de Cultura Económica.

ARIDA PÉRSIO (1984) "Neutralizar a inflação, uma idéia promissora". Economia e Perspectiva, Boletim do Conselho Regional de Economia, São Paulo, set.

"Múltiplos Equilíbrios" (2002) Revista de Economia Política, v. 22, n. 3, (87): 123131.

ARRIETA, G. M. G. (1988) "Interest rates, savings, and growth in LDC's: an assessment of Recent empirical research". World Development, v. 16, n. 5, p. 589-605.

BOGDANSKI, J., TOMBINI, A. A., WERLANG, S. R. C. (2000) "Implementing inflation targeting in Brazil", Working Paper Series, n. 1, Jul. Disponível em www.bcb.gov.br; acesso em 09/08/2004.

BRANSON, W. H. (1970) "Monetary policy and the new view of international capital movements", Brookings Papers on Economic Activity, n. 2, p. 235-270.

BRESSER-PEREIRA, L. C., NAKANO, Y. (2002) "Uma estratégia de desenvolvimento com estabilidade”, Revista de Economia Política, v. 22, n. 3, (87): 146-180.

CARDOSO, C. A., VIEIRA, F. V. (2004) "Câmbio, inflação e juros na transição do regime cambial brasileiro: uma análise de vetores auto-regressivos e causalidade”, In: XXXII Encontro Nacional de Economia, 2004, João Pessoa. Anais do XXXII Encontro Nacional de Economia. São Paulo: ANPEC, 1 CD Rom.

CIRNE DE TOLEDO, J. E. (2002) "Risco-Brasil: o efeito-Lula e os efeitos-Banco Central”, Revista de Economia Política, v. 22, n. 3, (87): 139-145.

COULBOIS, P., PRISSERT, P. (1974) "Forward exchange, short term capital flows and monetary policy”, De Economist, v. 122, n. 4, p. 283-308.

DIAZ-ALEJANDRO, C. (1985) “Good-bye financial repression, hello financial crash", Journal of Development Economics, n. 19, p. 1-24.

ENGLE, R. F., GRANGER, C. W. J. (1987) “Co-integration and error correction: representation, estimation, and testing”, Econometrica, v. 55, n. 2, p. 251-276.

FASOLO, A. M., PORTUGAL, M. S. (2003) "Imperfect rationality and inflationary inertia: a new estimation of the Phillips Curve for Brazil", Texto para discussão, UFRGS-PPGE. Disponível em: http//:www.ufgrs.br/ppge; acesso em 10/10/2004.

FERRARI FILHO, F., MENDONÇA, H. F. de, SOBREIRA, R. (2003) "Proposta de regime cambial para a economia brasileira”, In: J. Sicsú, J. L. Oreiro, L. F. de Paula (orgs.). Agenda Brasil: políticas econômicas para o crescimento com estabilidade de preços. São Paulo: Manole: Fundação Konrad Adenauer, p. 153-200. 
FERRARI FILHO, F. (2001-2) "Why does it not make sense to create a monetary union in Mercosur? A keynesian alternative proposal”, Journal of Post Keynesian Economics, v. 24, n. 2, p. 235-252.

FIGUEIREDO, F. M. R., FERREIRA, T. P. (2002) “Os preços administrados e a inflação no Brasil”. Trabalhos para discussão. Brasília, n. 59, 32 p. dez. 2002, Disponível em http://www.bcb. gov,br; acesso em 10/03/2004.

FRANKEL, J. A. (1992) “Measuring international capital mobility: a review”. American Economic Review, 82 (2): 197-202.

FUJII., CHINN, M. D. (2001) “Fin de Siècle Real Interest Parity”. Working Paper 7880, september 2001, National Bureau of Economic Research. Disponível em: http://www.nber.org/papers/w7880, acesso em 15/03/2004.

GARCIA, M. G. P. (2003) “Brazil in the $21^{\text {th }}$ century: how to escape to a high interest trap?", Texto para discussão n. 0466, PUC-Rio, mar. Disponível em: http://www.econ.puc-rio/ Txt2000.html; acesso em 05/03/2005.

GONÇALVES, C. E. S., GUIMARÃES, B. (2005) "Monetary policy and the exchange rate in Brazil”, september, 12 p, Disponível em: http://personal.lse.ac.uk/guimarae/copom.pdf; acesso em 22/10/2005.

GRANGER, C. W. J. (1969) "Investigating causal relations by econometric models and crossspectral methods”. Econometrica, v. 37, n. 3, p. 424-438.

GRANGER, C. W. J. (1988) “Causality, cointegration, and control”, Journal of Economic Dynamics and Control, North-Holland, n. 12, p. 551-559.

GRANGER, C. W. J., HUANG, B., YANG, C. W. (1998) “A bivariate causality between stock prices and exchange rates: evidence from recent Asia Flu", University of California, San Diego, Discussion Paper 98-09, april, 21 p. Disponível em: http://www.econ.ucsd.edu/papers/ files/ucsd9809.pdf; acesso em 21/06/2004.

HARVEY, J. T. (2004) "Deviations from uncovered interest rate parity: a post keynesian explanation”, Journal of Post Keynesian Economics, v. 27, n. 1, p. 19-35.

HOLANDA, M. C., CAVALCANTE, M. T. (2001) "Mobilidade de capital internacional no Brasil”, Revista de Economia Aplicada, São Paulo: FEA/USP-FIPE, v. 5, n. 2, p. 261-279.

KALDOR, N. (1982) The Scourge of Monetarism. Oxford: Oxford University Press.

KAM, E., SMITHIN, J. (2004) "Monetary policy and demand management for the small open economy in contemporary conditions whit (perfectly) Mobile Capital”, Journal of Post Keynesian Economics, v. 26, n. 4, p. 679-694.

LAVOIE, M. (1992) Foundations of Post-Keynesian Economic Analysis. Aldershot, UK: Edward Elgar.

(2000) "A post keynesian view of interest parity theorems", Journal of Post Keynesian Economics, v. 23, n. 1, p. 163-179.

“The Reflux Mechanism and the Open Economy”. In: L.-P. Rochon, M. Vernengo (eds.). Credit, interest rates, and the open economy. UK, Cheltenham: Edward Elgar, 2001, p. 215-242. 
(2002-3) "Interest parity, risk premia, and Post Keynesian analysis", Journal of Post Keynesian Economics, v. 25, n. 2, p. 237-249.

LOPES, F. L. (1985) "Inflação inercial, hiperinflação e desinflação: notas e conjecturas", Revista de Economia Política, v. 5, n. 2, p. 135-151.

MOORE, B. J. (1988) “The endogenous money supply", Journal of Post Keynesian Economics, v. 10, n. 3, p. 372-385.

(1991) "Money supply endogeneity: 'reserve price setting' or 'reserve quantity setting?", Journal of Post Keynesian Economics, v. 3, n. 3, p. 404-413.

NETO, A. F. C., VERNENGO, M. “Uma releitura heterodoxa de Bresser-Nakano", Revista de Economia Política, 2002, v. 22, n. 4, (88): 152-155.

NURKSE, R. (1944) International Currency Experience: lessons of inter-war period. Princeton: Princeton University Press, (Series of League of Nations).

OREIRO, J. L. (2002) "Prêmio de risco endógeno, metas de inflação e câmbio flexível: Implicações dinâmicas da hipótese Bresser-Nakano para uma pequena economia aberta", Revista de Economia Política, v. 22, n. 3, (87): 107-122.

PARASKEVOPOULOS, C. C., PASCHAKIS, J., SMITHIN, J. (1996) "Is monetary sovereignty an option for the small open economy?", North American Journal of Economics e Finance, v. 7, n. 1, p. 5-18.

PASCHAKIS, J., SMITHIN, J. (1998) "Exchange risk and the supply-side effects of real interest rate changes", Journal of Macroeconomics, v. 20, n. 4, p. 703-720.

PATTERSON, K. (2000) An introduction to applied econometrics: a times series approach. St. Martin's press.

PAULA, L. F., OREIRO, J. L., SILVA, G. J. C. (2003) "Fluxos e Controles de Capitais: avaliação e proposta de política”. In: J. Sicsú, J. L. Oreiro, L. F. de Paula (orgs.) Agenda Brasil: políticas econômicas para o crescimento com estabilidade de preços. São Paulo: Manole: Fundação Konrad Adenauer, 2003, p. 65-115.

ROCHA, K., MOREIRA, A. (2003) "Determinants of the brazilian sovereign risk: a two-factor structural model”, IPEA Discussion Paper n. 945, abril/2003. Disponível em: www.sbe. org.br/ebe25/136.pdf; acesso em 11/06/2004.

ROCHON, L. P., VERNENGO, M. (eds.) (2001) Credit, interest rates, and the open economy. UK, Cheltenham: Edward Elgar.

SICSÚ, J. (2002) "Flutuação cambial e taxa de juros no Brasil", Revista de Economia Política, 2002, v. 22 , n. 3 (87): 132-145.

SICSÚ, J., OREIRO, J. L., DE PAULA, L. F. (orgs.). (2003)Agenda Brasil: políticas econômicas para o crescimento com estabilidade de preços. São Paulo: Manole: Fundação Konrad Adenauer.

SILVA JR., A. F., SILVA, E. D. (2004) “Optimal international reserves holdings in emerging markets Economies: the Brazilian case”. In: XXXII Encontro Nacional de Economia, João Pessoa, PB. Anais do XXXII Encontro Nacional de Economia. São Paulo: ANPEC, 1 CD Rom. 
SMITHIN, J. (1997) "An alternative monetary model of inflation and growth", Review of Political Economy, 1997, v. 9, n. 4, p. 395-409.

(1999) "Money and national sovereignty in the global economy", Eastern Economic Journal, v. 25, n. 1, p. 49-61.

(2002-3) “Interest parity, purchasing power parity, 'risk premia', and Post Keynesian economic analysis”, Journal of Post Keynesian Economics, v. 2, n. 2, p. 219-235.

(2001) "Profit, the rate of interest, and 'entrepreneurship" in contemporary capitalism”, Kurswechsel, n. 2, p. 89-99.

(2003). Controversies in Monetary Economics. Cheltenham, UK: Edward Elgar.

The Economist, 23 de abril.

TVERSKY, A., KAHNEMAN, D. (1990) "Rational choice and the framing of decisions", In:

K. Cook, M. Levi, (eds.). The Limits of Rationality, Chicago: Chicago University Press, p. $60-89$.

VERNENGO, M. (2001) "Foreign Exchange, Interest and Prices: The Conventional Exchange Rate”, In: L.-P. Rochon, M. Vernengo (eds.), Credit, interest rates, and the open economy. UK, Cheltenham: Edward Elgar. p. 256-270.

VIEIRA, F., HOLLAND, M. (2003) "Country risk endogeneity, capital flows and capital controls in Brazil”, Revista de Economia Política, v. 23, n. 1 (89): 13-38.

WICKSELL, K. (1988) Lições de Economia Política. São Paulo: Nova Cultural.

WOODFORD, M. (2001) “The rule and optimal monetary policy”, American Economic Review, v. 91, n. 2, p. 232-237. 